Ciencias Agrarias/Agricultural Science

Cienc Tecn UTEQ (2018) 11(1) p 45-53 ISSN 1390-4051; e-ISSN 1390-4043

\title{
Caracterización de Sistemas de Producción Agropecuarios en el proyecto de riego Guarguallá-Licto, cantón Riobamba, provincia de Chimborazo
}

\section{Characteization of Agricultural production systems in the Guarguallá-Licto irrigation project, canton Riobamba, province of Chimborazo}

Victor Hugo Verdezoto Vargas*, Jorge Enrique Viera Pico

Universidad de Guayaquil, Facultad de Ciencias Agrarias. Guayaquil-Ecuador

\{victor.verdezotov, jorge.vierap\}@ug.edu.ec

*Autor para correspondencia: victor.verdezotov@ug.edu.ec

\begin{abstract}
Resumen
E sta investigación surge por la necesidad de identificar y caracterizar las tipologías campesinas y sistemas de producción agropecuarios predominantes en el proyecto de riego Guarguallá - Licto, ubicado en la parroquia Licto, cantón Riobamba, provincia de Chimborazo, cuyo objetivo general fue identificar los sistemas de producción agropecuarios, para la formulación de estrategias de intervención diferenciadas por sistema y tipología campesina, con lo cual se mejoraría los ingresos económicos de las comunidades. La población objetivo estuvo constituida por 900 familias campesinas, donde todas las unidades productivas agropecuarias (UPA) fueron escogidas al azar desarrollándose un muestreo aleatorio simple. Las encuestas, se aplicaron a 133 pequeños productores rurales de 14 comunidades, lo que permitió recopilar información relevante de 30 variables identificadas de las características agrosocioproductivas. Como resultado se obtuvo que el $87.22 \%$ de pequeños productores conforman el mayor grupo denominado Tipología 1, los mismos que se encuentran dentro del Sistema de producción 1. El 12.78\% se distribuye entre pequeños grupos que pertenecen a la Tipología y Sistema de Producción 2. La propuesta de intervención para estos dos Sistemas de Producción se enfoca en la unificación de parcelas dispersas y prácticas de agricultura sostenible.
\end{abstract}

Palabras clave: agricultura, campesino, comunidad, desarrollo, proyecto y sostenible.
Rec.: 14.03.2018. Acept: : 20.06.2018 Publicado el 2 de Julio de 2018

\begin{abstract}
$T$ his research arises from the need to identify and characterize the rural typologies and agricultural production systems predominant in the Guarguallá - Licto irrigation project, located in Licto parish, canton Riobamba, Chimborazo province. Its general objective was to identify the agricultural production systems for the formulation of intervention strategies differentiated by peasant system and typology, which would improve the economic income of the communities. The target population consisted of 900 peasant families, where all the agricultural productive units (UPA) were chosen at random, and a simple random sample was developed. The surveys were applied to 133 small rural producers in 14 communities, what allowed the collection of relevant information from 30 identified variables of agrosocioproductive characteristics. As a result, it was obtained that $87.22 \%$ of small producers make up the largest group called Typology 1 within Production System 1. The $12.78 \%$ is distributed among small groups that belong to Production System 2. The proposed intervention for these two production systems focuses on the unification of scattered plots and sustainable agriculture practices.
\end{abstract}

Keywords: agriculture, peasant, community, development, project and sustainable 


\section{Introducción}

$\mathrm{U}$ n sistema de producción es una combinación de diversos subsistemas; los de cultivo, definidos a nivel de las parcelas explotadas de manera homogénea, con las mismas tecnologías y sucesiones de siembras (en este sentido se distinguen varios sistemas agrícolas dentro de un proceso productivo); los de crianza, definidos a nivel de hatos o rebaños de animales; los de transformación de los productos agropecuarios (transformación de cereales, fabricación de quesos, etc.); y, las actividades no agrícolas (Apollin y Eberhart, 1999). El sistema de autosubsistencia es conocido como producción familiar ya que son ellos los que aprovechan sus recursos productivos (Palacios y Barrientos, 2014).

Los objetivos del desarrollo rural se enfocan básicamente en 4 aspectos: mejoramiento de capacidades económicas, humanas, políticas y de protección al medio ambiente. En relación a las económicas, se refiere al mejoramiento del ingreso por actividades agrícolas, por actividades diferentes a las agrícolas, mejoramiento de industrias y desarrollo de infraestructuras (BID, 2014). De acuerdo a Villa (2011), el desarrollo ha buscado la reducción de la pobreza y el mejoramiento de las condiciones de vida de la población más pobre. El incremento del nivel de vida incluye mejores ingresos, provisión de trabajo, acceso a educación, cultura y valores humanos.

Los sistemas silvopastoriles se han tornado muy importantes, debido a la necesidad de lograr un uso, manejo y aprovechamiento sostenible de los recursos naturales (Lok y Fraga, 2011). La actividad silvopastoril está dirigida a optimizar la producción agropecuaria, de este modo genera oportunidades de incrementar la producción de biomasa con mayor calidad, permite la mejora y conservación de suelos degradados, y hace los sistemas productivos más biodiversos (Murgueitio y Muhammad, 2010).

Los sistemas de producción agropecuaria enfrentan grandes desafíos debido a la globalización, siendo de gran relevancia que dichos sistemas mejoren su gestión para poder adaptarse a los diversos cambios del entorno y mantener un nivel de competitividad sustentable (Aguilera et al., 2003).

El sector agropecuario ecuatoriano constituye un pilar fundamental para el desarrollo económico del país. En efecto, la contribución de la agricultura al Producto Interno Bruto (PIB) nacional alcanzó valores superiores al 10\% (MAGAP, 2016). En este contexto, el riego es un medio trascendental para alcanzar esta cifra, y su aporte al PIB agrícola es todavía desconocido; sin embargo, diversos estudios resaltan su importancia para mejorar la productividad, generar empleo e incrementar los ingresos de las familias campesinas (Román y Gaybor, 2006).

Se considera que los sistemas de producción agroecológicos son capaces de restablecer y conservar el suelo, el agua y la biodiversidad en general. Por ello es necesario compararlos con otros sistemas y enfoques de producción teniendo en cuenta indicadores de sustentabilidad. (Astier, Masera y Galván, 2008)

Un sistema de agricultura sostenible está basado en normas de producción específicas y precisas cuya finalidad es lograr agroecosistemas óptimos que sean económicamente rentables, socialmente participativos y ecológicamente equilibrados. Ello, destacando el hecho de que las necesidades para el desarrollo de una agricultura sostenible no son solo ecológicas o técnicas, sino también socioculturales, económicas y políticas. (Lopez, 2007)

La selección de los sistemas de producción depende de los intereses que desarrolla cada productor, según la escasez relativa de los recursos disponibles con el fin de maximizar los ingresos por hectárea (Dufumier, 1990); generando interacciones entre la agricultura de subsistencia, la producción de cultivos comerciales y otros medios de diversificación de los ingresos (Katan et al., 2005). El agricultor de la región Litoral no está acostumbrado a manejar huertos caseros, por el contrario, prefiere el monocultivo ya que genera mayores ingresos económicos (Palacios y Barrientos, 2014).

La necesidad básica de alimentarse está negada para muchos. Por tanto, la investigación se convierte en la columna vertebral de la agricultura y compete a los responsables de las políticas y a los científicos a trabajar de común acuerdo para contribuir a reducir la pobreza, a compartir el crecimiento y a conseguir una distribución equitativa de los alimentos. (Sarageldin y Visser. 1999).

La necesidad de caracterizar los sistemas de producción en contextos de pobreza rural de América Latina, se debe a la gran diversidad de condiciones biofísicas y socioeconómicas diversas, pues la caracterización es fundamental para diseñar estrategias biodiversas, resilientes y socialmente justas para el consumo de la humanidad (Altieri y Nicholls, 2013). La estandarización y ponderación de indicadores, en función de procedimientos cuantitativos, permite la comparación entre fincas y el análisis de las múltiples dimensiones (ambiental, económica y social) de la sustentabilidad de los sistemas de producción (Sarandón et al., 2006). El Índice de Sustentabilidad de Sistemas Productivos Agrícolas (ISSPA) evalúa la integridad agropecuaria en el sistema productivo (Loaiza et al., 2014).

La superficie productiva total en la población en estudio es 1931 ha; de las cuales $31 \%$ corresponde a cebada, $18 \%$ a quinua y $12 \%$ a papa; el área regable es de 1357 ha, lo que representa el $70 \%$ de la superficie productiva (Coello et al., 2014).

Por la importancia que representa este estudio en el aporte al mejoramiento de las economías campesinas y nivel de vida de la población rural; esta investigación tuvo como objeto caracterizar los sistemas de producción y tipologías campesinas para definir estrategias de intervención diferenciadas por conglomerado. 


\section{Materiales y métodos}

Área de estudio

El estudio se realizó en la parroquia Licto, localizada a 13 km del cantón Riobamba, provincia de Chimborazo, república del Ecuador; limitada al norte por el canal de riego ChamboGuano, al sur por el río Guamote en su unión con el Cebadas, al este por el río Chambo y al Oeste con la parroquia Flores; ubicada dentro de las siguientes coordenadas geográficas: latitud $1^{\circ} 48^{\prime} 20^{\prime \prime} \mathrm{S}$, longitud $78^{\circ} 36^{\prime} 0^{\prime \prime} \mathrm{W}$ y altitud 2840 msnm (INAMHI, 2015).

De acuerdo a la ecología basada en las zonas de vida de Holdridge (1982), la parroquia Licto corresponde a las formaciones ecológicas estepa espinosa Montano Bajo (eeMB) y bosque seco Montano Bajo (bs-MB).

\section{Metodología}

L a población de estudio estuvo constituida por 900 familias campesinas, la mayoría de ellas indígenas en un $(96.3 \%)$ $\mathrm{y}$ un $3.7 \%$ mestizos localizados en la cabecera parroquial, pertenecientes a 14 comunidades ubicadas en 35 módulos pilotos, de las cuales 13 pertenecen a la parroquia Licto y una a la parroquia Flores; disponen de una extensión de 260 has regables en 4376 parcelas. Todas las unidades productivas agropecuarias (UPA) fueron escogidas al azar desarrollándose un muestreo aleatorio simple. Las encuestas, se aplicaron a 133 UPA, lo que permitió recopilar información relevante de las características agrosocioproductivas.

El tamaño de la muestra se determinó mediante la siguiente ecuación:

En donde:

$$
n=\frac{N P Q}{(N-1)\left(\frac{E}{K}\right)^{2}+P Q}
$$

$$
\begin{aligned}
& \mathbf{n}=\text { tamaño de la muestra } \\
& \mathbf{N}=\text { tamaño de la población } \\
& \mathbf{P}=\text { probabilidad de éxito } \\
& \mathbf{Q}=\text { probabilidad de fracaso } \\
& \mathbf{E}=\text { error } 8 \% \\
& \mathbf{K}=2 \text { (constante) } \\
& n=\frac{900(0.5)(0.5)}{(899)\left(\frac{0.08}{2}\right)^{2}+(0.5)(0.5)}=\frac{225}{1.69}=133
\end{aligned}
$$

\section{Datos registrados}

La información recopilada se realizó mediante encuestas de las unidades productivas agropecuarias, como género, ecología, poscosecha, consumo y comercialización. Además, se recopiló información sobre superficie de las unidades productivas, tenencia de la tierra, mano de obra, legalización de los predios, empleo, fuente de ingresos, migración, trabajo extra finca, crédito, precios, edad, escolaridad, miembros de la familia y actividades por miembro de familia. Las variables fueron clasificadas de acuerdo a sus propias características en grupos de familia, unidades productivas agropecuarias (UPA), producción y productividad y aspectos económicos; lo cual se explica detalladamente en Cuadro 1. 


\section{Cuadro 1. Clasificación de variables}

\begin{tabular}{|c|c|c|}
\hline Grupos & Variable & Características \\
\hline Familia & $\begin{array}{l}\text { V1 Número de miembros por familia } \\
\text { V2 Número de hijos mayores de } 18 \text { años } \\
\text { V3 Nivel de escolaridad del jefe de familia } \\
\text { V4 Ocupación principal del jefe de familia }\end{array}$ & $\begin{array}{l}\text { Hay un promedio de } 4 \text { personas por núcleo } \\
\text { familiar y } 2 \text { hijos mayores de } 18 \text { años } \\
\text { El jefe de familia es básicamente } \\
\text { agricultor, de baja escolaridad y producen } \\
\text { para el autoconsumo }\end{array}$ \\
\hline $\begin{array}{l}\text { Unidad } \\
\text { Productiva } \\
\text { Agropecuaria } \\
\text { (UPA) }\end{array}$ & $\begin{array}{l}\text { V5 Número de parcelas } \\
\text { V6 Numero de cultivos } \\
\text { V7 Superficie total de la UPA }\left(\mathrm{m}^{2}\right) \\
\text { V8 Superficie dedicada al cultivo de alfalfa }\left(\mathrm{m}^{2}\right) \\
\text { V9 Superficie dedicada al cultivo de maíz }\left(\mathrm{m}^{2}\right) \\
\text { V10 Superficie dedicada a otros cultivos }\left(\mathrm{m}^{2}\right)\end{array}$ & $\begin{array}{l}\text { Las familias }(87.22 \%) \text { tienen un promedio } \\
\text { de } 7.761 .85 \mathrm{~m}^{2} \text { y } 6.41 \text { parcelas separadas, } \\
1881.71 \mathrm{~m}^{2} \text { es cultivo de alfalfa, } 3368.71 \mathrm{~m}^{2} \\
\text { cultivo de maíz, } 2511.44 \mathrm{~m}^{2} \text { otros cultivos }\end{array}$ \\
\hline $\begin{array}{l}\text { Producción y } \\
\text { productividad }\end{array}$ & $\begin{array}{l}\text { V11 Rendimiento de alfalfa }(\mathrm{kg} / \mathrm{ha}) \\
\text { V12 Producción de alfalfa dedicada a la venta }(\mathrm{kg}) \\
\text { V13 Producción de alfalfa para autoconsumo }(\mathrm{kg}) \\
\text { V16 Rendimiento de maíz }(\mathrm{kg} / \mathrm{ha}) \\
\text { V17 Producción de maíz dedicada a la venta }(\mathrm{kg}) \\
\text { V18 Producción de maíz dedicada al autoconsumo }(\mathrm{kg}) \\
\text { V21 Número de bovinos } \\
\text { V22 Número de porcinos } \\
\text { V23 Número de ovinos } \\
\text { V24 Número de animales de especies menores }\end{array}$ & $\begin{array}{l}\text { Producción de alfalfa: venden } 3276.64 \mathrm{~kg} \mathrm{y} \\
\text { consumen } 7447.35 \mathrm{~kg} \\
\text { Producción de maíz: venden } 224.58 \mathrm{~kg} \mathrm{y} \\
\text { consumen } 163.93 \mathrm{~kg} \\
\text { Poseen } 2.59 \text { bovinos, } 1-2 \text { porcinos y } 21.61 \\
\text { especies menores. }\end{array}$ \\
\hline
\end{tabular}

$\begin{array}{ll}\text { V25 Valor de los animales (USD) } \\ \text { V14 Costo de producción de alfalfa (USD/ha) } \\ \text { V15 Beneficio obtenido de la alfalfa (USD/UPA) } \\ \text { V19 Costo de producción de maíz (USD/ha) } \\ \text { Aspectos } & \text { V20 Beneficio obtenido del maíz (USD/UPA) } \\ \text { económicos } & \text { V26 Costo de la producción pecuaria (USD) } \\ & \text { V27 Valor neto comercializado de productos } \\ & \text { agropecuarios UPA (USD) } \\ & \text { V28 Ingreso por actividades no agropecuarias (USD) } \\ & \text { V29 Ingreso debido a la migración (USD) } \\ & \text { V30 Valor económico de las especies Forestales (USD) }\end{array}$

Comercialización neta por año U\$D 717.12 por todas las actividades agropecuarias Ingresos insignificantes por otras actividades

Ingresos por venta de especies forestales U\$D 472.50 por familia por año. Su siembra se realiza en terrenos de baja fertilidad.

V29 Ingreso debido a la migración (USD)

30 Valor económico de las especies Forestales (USD)

\section{Análisis estadístico}

Con la información obtenida, se calculó: media aritmética, desviación típica, coeficiente de variación, se determinaron valores máximos, mínimos y los rangos correspondientes. Para la caracterización de los sistemas de producción y tipologías campesinas referidas en el Cuadro 4, se aplicó el análisis multivariado de conglomerados; considerando para el efecto las variables más relevantes (superficie de las unidades productivas, uso del suelo, rendimiento, costos y beneficios; número de bovinos, porcinos, ovinos, especies menores, migración; costo de la producción pecuaria; valor económico de la producción agropecuaria y especies forestales), cuyas variables estudiadas fueron agrupadas mediante categorías.

El agrupamiento de variables se realizó mediante métodos multivariados, para lo cual se utilizó el análisis de conglomerados o clúster, que permite agrupar a los productores de acuerdo a su tipología, similitud y a su vez posibilita sugerir alternativas diferenciadas. Con este procedimiento se identificó grupos homogéneos a partir de datos heterogéneos.

Con las variables generadas en el análisis factorial, se procedió a construir los clusters o agrupamientos resultantes del cociente de los factores para los autovalores (eigenvalues), calculados con el método de vinculación entre grupos con la medida de intervalo de la distancia euclidiana al cuadrado, lo cual sirvió para el análisis de componentes principales.

\section{Resultados}

\section{Caracterización de los sistemas de producción}

Para esta caracterización, se consideraron variables bioeconómicas, bioagronómicas y características intrínsecas de las Unidades Productivas Agropecuarias (UPA). Las estadísticas descriptivas de las variables seleccionadas para la tipificación de los productores, indican los valores máximos y mínimos, media y el desvío estándar como un indicador de la variabilidad de esas observaciones (Cuadro 2). 
Cuadro 2. Estadísticas descriptivas de las variables seleccionadas para la tipificación de los productores

\begin{tabular}{|c|c|c|c|c|c|}
\hline Grupos & Variables & $\begin{array}{c}\text { Valor } \\
\text { Mínimo }\end{array}$ & $\begin{array}{c}\text { Valor } \\
\text { Máximo }\end{array}$ & $\begin{array}{c}\text { Media } \\
(n=133)\end{array}$ & $\begin{array}{l}\text { Desviación } \\
\text { Estándar }\end{array}$ \\
\hline \multirow{5}{*}{ Familias } & \multirow{5}{*}{$\begin{array}{ll}\text { V1 } & \text { Número de miembros po familia } \\
\text { V2 } & \begin{array}{l}\text { Número de hijos mayores } \\
\text { de } 18 \text { años }\end{array} \\
\text { V3 } & \begin{array}{l}\text { Nivel de escolaridad del jefe } \\
\text { de familia }\end{array} \\
\text { V4 } \begin{array}{l}\text { Ocupación principal del jefe } \\
\text { de familia }\end{array}\end{array}$} & 2.00 & 13.00 & 5.87 & 2.33 \\
\hline & & 0.00 & 9.00 & 1.71 & 1.99 \\
\hline & & & & & \\
\hline & & 1.00 & 7.00 & 2.43 & 1.14 \\
\hline & & 1.00 & 6.00 & 1.84 & 1.71 \\
\hline \multirow{7}{*}{$\begin{array}{l}\text { Unidad } \\
\text { Productiva } \\
\text { Agropecuaria } \\
\text { (UPA) }\end{array}$} & \multirow{7}{*}{$\begin{array}{cl}\text { V5 } & \text { Número de parcelas } \\
\text { V6 } & \text { Número de cultivos } \\
\text { V7 } & \text { Superficie total de la UPA }\left(\mathrm{m}^{2}\right) \\
\text { V8 } & \begin{array}{l}\text { Superficie dedicada al cultivo } \\
\text { de alfalfa }\left(\mathrm{m}^{2}\right)\end{array} \\
\text { V9 } & \begin{array}{l}\text { Superficie dedicada al cultivo } \\
\text { de maíz }\left(\mathrm{m}^{2}\right)\end{array} \\
\text { V10 Superficie dedicada a otros } \\
\text { cultivos }\left(\mathrm{m}^{2}\right)\end{array}$} & 2.00 & 25.00 & 6.61 & 3.79 \\
\hline & & 1.00 & 8.00 & 3.57 & 1.39 \\
\hline & & 363.00 & 66000.00 & 8780.41 & 9638.99 \\
\hline & & & & & \\
\hline & & 0.00 & 15000.00 & 2278.47 & 2961.22 \\
\hline & & 0.00 & 34000.00 & 3649.34 & 4752.88 \\
\hline & & 0.00 & 36000.00 & 2852.61 & 4470.47 \\
\hline \multirow{10}{*}{$\begin{array}{l}\text { Producción y } \\
\text { productividad }\end{array}$} & \multirow{2}{*}{$\begin{array}{l}\text { V11 Rendimiento de alfalfa (kg/ha) } \\
\text { V12 Producción de alfalfa dedicada } \\
\text { a la venta (kg) } \\
\text { V13 Producción de alfalfa dedicada } \\
\text { al consumo en la }\end{array}$} & 0.00 & 79375.00 & 54461.59 & 17213.29 \\
\hline & & 0.00 & 80962.50 & 4341.25 & 9411.92 \\
\hline & UPA $(\mathrm{kg})$ & 0.00 & 76200.00 & 8375.38 & 11376.62 \\
\hline & V16 Rendimiento de maíz (kg/ha) & 0.00 & 3024.00 & 1188.49 & 536.98 \\
\hline & V17 Producción de maíz dedicada & 0.00 & 4536.00 & 258.63 & 515.91 \\
\hline & $\begin{array}{l}\text { V18 Producción de maíz dedicada } \\
\text { al autoconsumo }(\mathrm{kg})\end{array}$ & 0.00 & 989.68 & 173.33 & 180.55 \\
\hline & V21 Número de bovinos & 0.00 & 8.00 & 2.59 & 1.65 \\
\hline & V22 Número de porcinos & 0.00 & 10.00 & 1.71 & 1.58 \\
\hline & V23 Número de ovinos & 0.00 & 11.00 & 2.50 & 2.45 \\
\hline & $\begin{array}{l}\text { V24 Número de animales de especies } \\
\text { menores }\end{array}$ & 0.00 & 81.00 & 24.03 & 16.26 \\
\hline \multirow{11}{*}{$\begin{array}{l}\text { Aspectos } \\
\text { económicos }\end{array}$} & \multirow{11}{*}{$\begin{array}{l}\text { V25 Valor de los animales (USD) } \\
\text { V14 Costo de producción de alfalfa } \\
\text { (USD/ha) } \\
\text { V15 Beneficio obtenido del cultivo } \\
\text { de alfalfa (USD/UPA) } \\
\text { V19 Costo de producción de maíz } \\
\text { (USD/ha) } \\
\text { V20 Beneficio obtenido del cultivo } \\
\text { del maíz (USD/UPA) } \\
\text { V26 Costo de la producción pecuaria } \\
\text { (USD) } \\
\text { V27 Valor neto comercializado de } \\
\text { productos agropecuarios UPA (USD) } \\
\text { V28 Ingreso debido a actividades no } \\
\text { agropecuarias (USD) } \\
\text { V29 Ingreso debido a la migración (USD) } \\
\text { V30 Valor económico de las especies } \\
\text { Forestales (USD) }\end{array}$} & 101.00 & 2410.00 & 701.11 & 439.58 \\
\hline & & 0.00 & 996.67 & 400.22 & 222.94 \\
\hline & & 0.00 & 3974.00 & 488.10 & 625.27 \\
\hline & & & & & \\
\hline & & 0.00 & 1082.29 & 233.62 & 192.78 \\
\hline & & 0.00 & 1978.00 & 124.21 & 210.18 \\
\hline & & 63.00 & 1628.00 & 680.34 & 310.76 \\
\hline & & 41.60 & 6647.00 & 839.12 & 1041.34 \\
\hline & & 0.00 & 500.00 & 39.29 & 85.25 \\
\hline & & 0.00 & 950.00 & 132.17 & 184.93 \\
\hline & & 0.00 & 50008.50 & 964.79 & 4692.74 \\
\hline
\end{tabular}


En el Cuadro 3, se reporta el análisis factorial de las variables definitivamente seleccionadas para la caracterización de los productores agropecuarios, pudiéndose observar que el conjunto de los seis primeros seleccionados explica el 70.83\% de la varianza total original.

El agrupamiento de los productores se realizó mediante métodos multivariados, en este caso utilizando el análisis de conglomerados o CLUSTER, que permite agrupar a los productores de acuerdo a su tipología, es deci, conforme a su similitud a partir de variables que fueron tomadas en la muestra y a su vez posibilita sugerir alternativas diferenciadas de acuerdo a los grupos de productores. En este caso, cada tipología corresponde a su propio sistema de producción, porque las características de las variables identificadas aseveran aquello. Con este procedimiento se identificaron grupos relativamente homogéneos a partir de variables heterogéneas, basándose en las características seleccionadas.

En el Cuadro 4, a partir de los datos de los productores, se identificaron seis conglomerados, en los que se muestra el número de casos por agrupamiento y su equivalencia en porcentaje respecto al total de productores estudiados, indicándose que un conglomerado corresponde a un sistema de producción y/o tipología campesina.

Cuadro 3. Varianza total explicada mediante el método de análisis de componentes principales.

\begin{tabular}{cccccccccc}
\hline & \multicolumn{3}{c}{ Auto valores iniciales } & \multicolumn{2}{c}{$\begin{array}{c}\text { Suma de las saturaciones } \\
\text { al cuadrado de la extracción }\end{array}$} & \multicolumn{2}{c}{$\begin{array}{c}\text { Suma de las saturaciones } \\
\text { al cuadrado de la rotación }\end{array}$} \\
\cline { 2 - 10 } \\
\cline { 2 - 10 }
\end{tabular}

Cuadro 4. Número de casos por agrupamiento y su equivalencia en porcentaje

\begin{tabular}{lcr}
\hline $\begin{array}{l}\text { Clúster / Conglomerado } \\
\text { (Sistema de Producción) }\end{array}$ & Número de casos por clúste & Porcentaje (\%) \\
\hline 1 & 116 & 87.22 \\
2 & 9 & 6.77 \\
3 & 3 & 2.26 \\
4 & 1 & 0.75 \\
5 & 3 & 2.26 \\
6 & 1 & 0.75 \\
Total & 133 & 100.00 \\
\hline
\end{tabular}

\section{Sistema de Producción 1}

El $87.22 \%$ de productores pertenecientes a esta tipología conforman este sistema de producción, tienen similitud respecto a la disponibilidad de recursos tecnológicos y económicos, manejan pocos recursos y dinero, por lo que cualquier futura intervención debe considerar a este grupo como el más importante. Hay un promedio de 4 personas por núcleo familiar y 2 hijos mayores de 18 años, el jefe de familia es básicamente agricultor, de baja escolaridad y producen para el autoconsumo.

Las características productivas de esta tipología son las de disponer de un promedio de 6.41 parcelas por familia en una superficie de $7761.85 \mathrm{~m}^{2}$; de las cuales $1881.71 \mathrm{~m}^{2}$ dedican al cultivo de alfalfa, $3368.71 \mathrm{~m}^{2}$ al cultivo de maíz y apenas $2511.44 \mathrm{~m}^{2}$ a otros cultivos; en relación a la producción de alfalfa $3276.64 \mathrm{~kg}$ lo dedican a la venta y $7447.35 \mathrm{~kg}$ para consumo, con un costo de producción de $374.49 \mathrm{USD} / \mathrm{ha}$, alcanzando un beneficio neto de 394.41 USD/UPA.

Respecto al cultivo de maíz, destinan $224.58 \mathrm{~kg}$ para la venta y $163.93 \mathrm{~kg}$ para autoconsumo, con un costo de producción de 235.27 USD/ ha, alcanzando un beneficio neto de 105.01 USD/ UPA. Disponen en promedio 2.59 bovinos y 21.61 animales menores, siendo 717.12 USD el total de la comercialización neta por año.

Para este tipo de familias, su situación se ve agravada por el hecho de que las parcelas generalmente se encuentran a distancias equidistantes (dispersas) entre sí. Con los pocos recursos que estas familias disponen sería prácticamente imposible pasar de una agricultura de subsistencia a otra enfocada al mercado, a menos que pudieran sembrar cultivos altamente rentables y de aceptable productividad.

Dadas las condiciones en las que se desarrolla la producción para este conglomerado, lo mejor sería optimizar los recursos disponibles con el propósito de asegurar su sustentabilidad, es 
decir utilizar variedades o material genético nativo de maíz y alfalfa que han demostrado excelente adaptabilidad a las condiciones locales, combinando con prácticas agroecológicas de manejo del suelo.

\section{Sistema de Producción 2}

Este conglomerado que corresponde a la tipología 2 representa el $6.77 \%$ del total de familias caracterizadas. Disponen de una superficie promedio de $13921.62 \mathrm{~m}^{2}$, en 8.33 parcelas; la superficie dedicada al cultivo de alfalfa es de $3755.22 \mathrm{~m}^{2}$, $7110.22 \mathrm{~m}^{2}$ al maíz y $3056.22 \mathrm{~m}^{2}$ a otros cultivos.

Dentro de este sistema existe una producción promedio de $6928.73 \mathrm{~kg}$ de alfalfa destinada a la venta y $13035.31 \mathrm{~kg}$ para autoconsumo, con un costo de producción de 615.96 USD/ ha, dando un beneficio neto de 1076.87 USD/UPA. Venden $718.20 \mathrm{~kg}$ de maíz y dedican al autoconsumo $277.20 \mathrm{~kg}$; el costo de producción de maíz es de $186.74 \mathrm{USD} / \mathrm{ha}$, con un beneficio neto de 342.63 USD/UPA.

En la unidad productiva agropecuaria, tienen un promedio de 1.56 bovinos y 28 animales menores. El valor total de la producción agropecuaria comercializada es de 1757.78 USD/ UPA. Dentro de las UPAs el valor estimado de las especies forestales es de 2091.06 USD/UPA.

Los sistemas de producción 1 y 2, que poseen tipologías campesinas semejantes, representan el $93.99 \%$ de familias estudiadas, por lo tanto, los restantes agrupamientos $(3,4,5 \mathrm{y}$ 6 , ver Tabla 3), en conjunto apenas significan el 6.01\%, éstos conglomerados disponen de mayores y mejores recursos. Por razones lógicas, las estrategias de intervención en estos grupos o tipologías (clusters 3, 4, 5 y 6), deben ser similares a las que se apliquen al sistema de producción 2, enfatizando en la posibilidad de insertarlos a mercados regionales con productos de calidad y que sean capaces de agregar valor a su producción primaria, diversificando su producción, de acuerdo a sus propias características.

Organizativamente, estas familias deberían estar asociadas de acuerdo a sus actividades productivas, con la posibilidad de sentar las bases para fomentar sistemas de cultivo y crianza de rebaños que puedan insertarse en los mercados más competitivos de la región y a la vez puedan reducir o eliminar el número de intermediarios en la cadena de valor de sus productos.

\section{Discusión}

E n la parroquia Licto, el número promedio de miembros por familia es de 3.8 (INEC, 2010b). Las familias estudiadas tienen en promedio 1.7 hijos mayores de 18 años, con un desvío estándar de \pm 2.0 (Verdezoto, 2005), lo cual indica que siempre 4 miembros de la familia (padre, madre e hijos mayores de edad) aportan al trabajo productivo familiar. En relación del jefe o jefa de hogar, el 58\% son hombres y el $42 \%$ mujeres (Coello et al., 2014); sin embargo, en el sector rural se considera que las mujeres contribuyen y aportan al trabajo productivo familiar. Respecto a la edad de los hijos, en el estrato comprendido entre 11 a 20 años, se encuentra el $37.7 \%$ de la población, con un promedio de 16.5 años y una desviación estándar de \pm 9.6 ; esta población es parte del grupo familiar dedicada a estudios secundarios y de tercer nivel.

En cuanto a la ocupación principal de los miembros de las familias, el 54\% son mujeres dedicadas a la actividad agropecuaria (Coello et al., 2014), la agricultura y alguna otra actividad económica desplegada simultáneamente son desarrolladas por el $12 \%$ de personas migrantes (INEC, 2010a). Al igual que en otros lugares del sector rural, se puede apreciar una activa participación de las mujeres, según Bocero y Di Bona, (2012), el trabajo femenino asalariado está presente en la actividad hortícola que se practica en el campo. Los indicadores de desarrollo en economías campesinas permiten la valoración de características básicas de sustentabilidad, pero también tienen la capacidad de reflejar las particularidades de los sistemas de producción, que son diversos, pero siempre multidimensionales y dinámicos. En la práctica, no existe un listado general de indicadores, estos podrían clasificarse por ser de tipo ambiental, económico, social o político, pero tienen que responder a un criterio de diagnóstico asociado a la valoración de alguno de los atributos de sustentabilidad (Pérez et al., 2016), que comparado con el estudio realizado se presentan algunas semejanzas en indicadores identificados.

Los sistemas de producción sustentables son aquellos que permanecen estables en el tiempo, por lo cual poseen la capacidad de regenerarse, autorregularse y transformarse, para que el sistema sea sostenible (Astier et al., 2008). Los sistemas de producción agropecuaria se encuentran expuestos a riesgos de orden ambiental y sociocultural; alcanzar su sustentabilidad constituye un desafío creciente para la gestión de los gobiernos que en sus políticas públicas buscan salir del paradigma del desarrollismo clásico, basado en crecimiento económico ilimitado a costa de la sobreexplotación de los recursos naturales y la desigualdad social (Álvarez y Zulaica, 2015). La tipificación busca agrupar a productores con características similares de manejo y producción agropecuaria, en cuyo caso derivan a un tipo de sistema productivo (Rocha et al., 2016), lo cual concuerda precisamente con la identificación de los sistemas de producción y tipologías campesinas identificadas y que al aplicarse prácticas resilientes con el ambiente, los sistemas por sí solos tenderán autorregularse en el tiempo. Como resultado del estudio, se considera que el aumento permanente de la formación de capital en la mayoría de las familias de productores agrícolas es una de las prioridades de la agricultura familiar y que con la intervención de los actores locales y el auspicio de las entidades gubernamentales, para proporcionar bienes, activos y servicios que contribuyan permanentemente, aportando a la seguridad alimentaria y al bienestar de la población rural, teniendo en cuenta la competitividad, mercados, recursos naturales, diversidad biológica, con actitud sostenible y sustentable, que aporten a los objetivos del buen vivir. 


\section{Conclusiones}

\section{Sistema de Producción I}

Implementar una metodología que permita que los lotes de terreno de la misma superficie, sean intercambiados entre productores interesados, de tal manera que se dispongan de manera cercana entre sí y de esa forma se pueda mejorar la eficiencia de los sistemas productivos.

Con la aplicación de tecnologías limpias (agricultura sustentable), se puede elevar los rendimientos sobre todo de alfalfa y maíz; de esa forma habrá mayor posibilidad de consumir dentro de la unidad productiva y comercializar sus excedentes productivos.

Hacer uso de los recursos disponibles del medio (agua de riego, materia orgánica, semillas andinas) para apuntar hacia la sustentabilidad de este sistema de producción.

Mejorar la genética de bovinos y especies menores y su nutrición para lograr mayores rendimientos biológicos $\mathrm{y}$ económicos.

Dentro del concepto de agricultura sostenible, se debería incorporar la plantación de especies forestales nativas, para establecer sistemas agroforestales.

\section{Sistema de Producción II}

La disponibilidad de recursos de estos grupos, podría permitir la implementación de una agricultura comercial, que considere desde la planificación, hasta la negociación, permitiendo agregar valor a la producción primaria.

Tecnológicamente con los productores que pertenecen a este sistema, se debería fomentar esfuerzos para desarrollar y consolidar una visión productiva basada en la agricultura sostenible, que permita incrementar los rendimientos y manejar de manera sustentable los recursos naturales.

Los productores de estos grupos deberían capacitarse ampliamente en aspectos de manejo y sanidad animal, pues su potencial de producción animal puede ser una alternativa para incrementar sus ingresos monetarios.

Se recomienda por razones lógicas que, dentro de sus actividades productivas, se incluyan a los clústeres $3,4,5$ y 6 , por constituir tipologías y sistemas de producción de pocos productores y con mejores recursos e ingresos.

\section{Bibliografía}

Aguilera M, Bruna G, Brzovic F, Cerda R, Clark M, Chandía A, Domínguez J I, Espinoza A, Faúndez M, García P, Jara C, De Kartzow A, Kern W, Lerdón J, Marchant R, Mora M, Olavarría J, Paillacar R, Quijada A, Troncoso J L y Vargas G. (2003). Fundamentos de gestión para productores agropecuarios: tópicos y estudios de casos consensuados por universidades chilenas. Programa. Santiago de Chile.

Altieri, M. y Nicholls, C. (2013). Agroecología : Única esperanza para la soberanía alimentaria y la resiliencia socioecologica, 7(2), 65-83

Astier, M., Masera, O. y Galván, Y. (2008). Evaluación de la Sustentabilidad. Un enfoque dinámico y multidimensional. Recuperado de http://docplayer. es/14885206-Evaluacion-de-sustentabilidad-un-enfoquedinamico-y-multidimensional.html.

Álvarez, S. y Zulaica, L. (2015). Indicadores de sustentabilidad en sistemas de albarradas: aportes metodológicos. Letras Verdes Revista Latinoamericana de Estudios Socioambientales 18, 184-207. doi: 10.17141/ letrasverdes.18.2015.1634

Apollin, F. y Eberhart, C. (1999). Análisis y diagnóstico de los sistemas de producción en el medio rural. Guía metodológica. Ecuador: Quito, 237

Astier, M., Masera, O. y Galván, Y. (2008). Evaluación de la Sustentabilidad. Un enfoque dinámico y multidimensional. Recuperado de http://docplayer. es/14885206-Evaluacion-de-sustentabilidad-un-enfoquedinamico-y-multidimensional.html.

Banco Interamericano de Desarrollo (2014), Política de desarrollo rural. Consultado marzo 10, 2014 de Banco Interamericano de Desarrollo. Web Site: http://www.iadb. org/es/acerca-del-bid/politica-dedesarrollo-rural,6229. html

Bocero, L. S. y Di Bona, A. (2012). El trabajo asalariado femenino en el cinturón frutihortícola marplatense. Geograficando, 8(8), 81-101

Coello, P., Lobato, M., Riofrío, R., Guallpa, P. y Guevara, I. (2014). Plan de Desarrollo y Ordenamiento Territorial 2015-2019. Recuperado de: http://www.licto.gob. ec/index.php/gad-licto/plan-de-desarrollo/1-plan-dedesarrollo-y-ordenamiento-territorial/file

Dufumier, M. (1990). Importancia de tipología de unidades de producción agrícolas en el análisis de diagnostico de realidades agrarias. En J. Berdegué, M. Dufumier y G. Escobar. Tipificación de Sistemas de Producción Agricola. (pp. 63-81). Chile: Santiago de Chile. Recuperado de: https://doi.org/ISBN. 956.7110-01-07

Holdridge, L. (1982). Ecología basada en zonas de vida (1a ed.). Costa Rica: San José. Recuperado de: https:// books.google.es/books?hl=es\&lr=\&id=m3Vm2TCjM MC\&oi =fnd\&pg=PR9\&dq=holdridge\&ots $=$ oMfDu2IwH\&sig=tPN-C530xrtV2j3w2udskLfh R9M\#v=one page $\& \mathrm{q}=$ holdridge $\& \mathrm{f}=$ false

INAMHI (2015). Anuario meteorológico № 52-2012. Quito - Ecuador. Recuperado de: http://www. serviciometeorologico.gob.ec/wpcontent/uploads/ anuarios/meteorologicos/Am\%202012.pdf

INEC (2010a). Poblacion migrante por sexo, según provincia, cantón y parroquia de empadronamiento. Recuperado de: http:/www.ecuadorencifras.gob.ec/informacion-censalcantonal/

INEC (2010b). Promedio de personas por hogar a nivel Nacional. Recuperado de: http:/www.ecuadorencifras. 
gob.ec/informacion-censal-cantonal/

Katan, C., Terenziani, F., Mongin, O., Werts, M., Porres, L., Pons, T., Mertz, J., Tretiak, S. y Blanchard, M. (2005). Effects of (Multi)branching of Dipolar Chromophores on Photophysical Properties and Two-Photon Absorption. The Journal of PHYSICAL CHEMISTRY A. 109 (13), 3024-3037. doi: 10.1021/jp044193e. https://doi. org/10.1021/jp044193e.

Loaiza, W., Carvajal, Y. y Ávila, Á. J. (2014). Evaluación agroecológica de los sistemas productivos agrícolas en la microcuenca Centella (Dagua, Colombia). Colombia Forestal, 17(2), 161-179. https://doi.org/10.14483/ udistrital.jour.colomb.for.2014.2.a03

Lok, S. y S. Fraga. "Comportamiento de indicadores del suelo y del pastizal en un sistema silvopastoril de Leucaena leucocephala / Cynodon nlemfuensis con ganado vacuno en desarrollo". Revista Cubana de Ciencia Agrícola, Vol. 45(2011): 195-202

Lopez, R. C. (2007). Sistemas d eproduccion agricola sostenible en los Andes de Venezuela: Agricultura. Avances de quimica, 23-33. doi:1856-5301

MAGAP. (2016). La política agropecuaria ecuatoriana: hacia el desarrollo territorial sustentable: 2015-2025 I parte. Ecuador: Ministerio de Agricultura, Ganadería, Acuacultura y Pesca.

Murgueitio, E. y I. Muhammad. "Agroforestería pecuaria para la reconversión de la ganadería en Latinoamérica". Livestock Res. Rural Development. Vol. 13 (2010).

Palacios, V. y Barrientos, J. (2014). Caracterización técnica y económica de los agrosistemas de producción en dos resguardos indígenas del Putumayo ( Colombia ).
Acta agronómica, 63(2), 91-100. doi: 10.15446/acag. v63n2.29358

Pérez, M., Ávila, A. y Torres, A. (2016). Evaluación de la sustentabilidad: una reflexión a partir del caso de la Red Nacional de Desarrollo Rural Sustentable (México). Entreciencias 4(9): 61-72. http://www.redalyc.org/ articulo.oa?id=457645340005

Rocha, C., Mora, J. y Romero, J. (2016). Tipología de sistemas de producción en la zona rural del municipio de Ibagué, Colombia. Agronomía Mesoamericana 27(2), 253-264. doi: 10.15517/am.v27i2.24360

Román, P., Gaybor, A. (2006). Impactos del riego en los sistemas de producción de Licto, Chimborazo, 2006. Ecuador: Universidad Central del Ecuador. Facultad de Ciencias Agrícolas.

Sarageldin, I., y Visser M. Food: nature and culture. Social Research 1999, vol 66, p. 103

Sarandón, S., Zuluaga, M., Cieza, R., Gómez, C., Janjetic, L. y Negrete, E. (2006). Evaluación de la sustentabilidad de sistemas agrícolas de fincas en misiones, argentina, mediante el uso de indicadores. Agroecología, 1, 19-28.

Verdezoto, V. (2005). Caracterización de los sistemas de produccion agropecuarios y propuesta de intervención en las comunidades bajo la influencia del proyecto de riego Guargualla - Licto, cantón Riobamba (Tesis de Maestría). Escuela Superior Politecnica de Chimborazo. Recuperado de: http://dspace.espoch.edu.ec/bitstream/123456789/ 4071/1/20T00096.pdf

Villa, I. M. R. (2011) ¿Qué hacemos con el campo? Mundiprensa México. Segunda Edición, pp 10- 20. 